\title{
Espacio íntimo de la pedagogía: relación educativa y su triple dimensión formativa como dinamismo de ciudadanía
}

\section{The intimate space of pedagogy: educational relationships and its triple formative dimension as citizenship dynamism}

\author{
Mónica Gijón Casares ${ }^{1}$ \\ mgijon@ub.edu \\ Universidad de Bacelona, España
}

\section{Resumen:}

En contextos de prostitución el espacio íntimo de la pedagogía constituye una forma de entrar en relación, construir un espacio común e impulsar a la emancipación. Cuando una mujer que ejerce la prostitución llega a una entidad social necesita una acogida cálida, pero su experiencia formativa está sujeta al clima de comprensión y reconocimiento que vive en el centro.

La relación es un dinamismo de humanización y formación que muestra la necesaria interdependencia de la vida humana (Lévinas, 2000; Noddings, 2009). Sin embargo, en situaciones de exclusión, no nos referimos a ella como dinamismo formativo, más bien reducimos su dimensión educativa a los modos de acompañamiento que requieren una cierta distancia profesional.

El trabajo que presentamos muestra que la relación es un dinamismo pedagógico nuclear que atraviesa y dota de sentido la cultura pedagógica de las entidades socia-

\begin{abstract}
:
In contexts of prostitution, the intimate space of pedagogy is a way of engaging, building a common space and promoting emancipation. When a prostitute comes into contact with a social entity she needs a warm welcome, but her training experience is subject to the climate of understanding and recognition that she might find in the education centre.

Relationships are humanising and training forms of dynamism that show the interdependence of human life (Lévinas, 2000; Noddings, 2009). However, in situations of exclusion, we substitute the educational dimension inherent in relationships with the modes of attachment and accompaniment typical of professional distance. The study we present shows that relationships contain a great pedagogical dynamism that crosses and fills with meaning the pedagogical culture of social entities. Relationships constitute a formative way that has a triple dimension: encounter, alliance and emancipation. This triple di-
\end{abstract}

1 Dirección para correspondencia (correspondence address):

Mónica Gijón Casares. Dept. Teoria i Història de l'Educació. 3er pis- Edifici de Llevant. Facultat d'Educació. Campus Mundet. Pg Vall d'Hebrn, n 171. 08035, Barcelona (España). 
Espacio íntimo de la pedagogía: relación educativa y su triple dimensión formativa como dinamismo de ciudadanía

Mónica GiJón CASARes

les. Una vía formativa que posee una triple dimensión de encuentro, alianza y emancipación visible en las formas de acogida y compromiso, en la voluntad de poner en valor a la mujer e impulsarla a un proceso de liberación individual y colectivo.

\section{Palabras clave:}

Relación educativa; encuentro; alianza; emancipación. mension is made visible in the forms of welcome and commitment, in the desire to value women and in the promotion of a process of individual and collective liberation.

\section{Keywords:}

Educational relation; meeting; alliance; emancipation.

\section{Résumé:}

Dans les contextes de prostitution, l'espace intime de la pédagogie est une manière d'entrer en relation, de construire un espace commun et de promouvoir l'émancipation. Lorsqu'une femme qui pratique la prostitution arrive à une entité sociale, elle a besoin d'un accueil chaleureux, mais son expérience formatrice est soumise au climat de compréhension et de reconnaissance qui habite l'institution.

La relation est un dynamisme d'humanisation et de formation qui montre l'interdépendance nécessaire de la vie humaine (Lévinas, 2000, Noddings, 2009). Cependant, dans les situations d'exclusion, nous ne nous référons pas à lui comme un dynamisme formateur, nous réduisons plutôt sa dimension éducative aux modes d'attachement et d'accompagnement qui requièrent une certaine distance professionnelle.

Le travail que nous présentons montre que la relation est un dynamisme pédagogique qui traverse et donne un sens à la culture pédagogique des institutions sociales. Une voie de formation qui a une triple dimension de rencontre, d'alliance et d'émancipation visible dans les formes de réception et d'engagement, dans la volonté de valoriser la femme et de la pousser à un processus de libération individuelle et collective

\section{Mots clés:}

Relation éducative; rencontre; alliance; émancipation.

Fecha de recepción: 1-6-2018

Fecha de aceptación: 7-10-2018

\section{La relación humana, el ámbito más íntimo de la pedagogía}

La propuesta que aquí presentamos es una aproximación al espacio más íntimo de la pedagogía, una descripción de la relación educativa y sus características como dinamismo formativo. El trabajo etnográfico en contextos de prostitución realizado en diferentes proyectos de atención a mujeres nos ha permitido detectar una triple naturaleza formativa de la relación que se concreta en tres dimensiones, el encuentro, la alianza y la emancipación. Una naturaleza formativa que se aprecia tanto en las formas de acogida y trato digno que dispensan las educadoras a las mujeres que atienden, como en los lazos de compromiso y mutualidad que nutren los procesos formativos. Todos ellos requieren de una vía de 
humanización y reconocimiento que se pone en juego en el espacio íntimo de la pedagogía.

La relación es una importante vía de recuperación y apoyo en los procesos de inclusión, sin embargo, los equipos educativos difícilmente se refieren a ella como una herramienta formativa, veamos algunas de las razones que quizá lo explican. Por un lado, la relación ha quedado desdibujada bajo la noción acompañamiento social, que se sitúa al profesional como un experto del "estar cerca" para ofrecer un espacio de aliento y ayuda (Planella, 2016). Aspecto sin duda esencial en contextos de exclusión, pero que no explica la complejidad y riqueza de la relación en todas sus dimensiones.

Por otro lado, la relación ha quedado reducida a una óptica subjetivista cuando se limita su potencial al carácter y la personalidad del educador. Aspecto sin duda importante, pero que deja a la pedagogía en una suerte de relativismo que deposita el éxito de un trayecto formativo en el carisma del educador. Finalmente, el análisis de las formas de poder ha contribuido a invisibilizar el potencial formativo de las relaciones debido en parte a que, desde ciertas ópticas críticas, parece imposible referirse a la relación sin en escapar al control -más o menos consciente- de los intereses autoritarios de un educador (Foucault, 2010).

La idea de acompañamiento, el papel del carisma y el análisis de poder deben ser tenidos en cuenta en el análisis pedagógico de las relaciones, sin embargo, dichas ópticas no explican el contenido formativo de la relación ni sustituyen su potencial como vía de humanización y reconocimiento. En ese espacio íntimo de la pedagogía, sus protagonistas ponen en juego aprendizajes, actitudes y grandes dosis de sensibilidad pedagógica que dan sentido a la formación. Sin embargo, no solo ponen en juego emociones y actitudes, los protagonistas se ponen en juego a sí mismos y lo hacen mutuamente por medio de la acción y el diálogo. Acercarnos a la relación en contextos de exclusión nos ha permitido comprender que, en el espacio más íntimo de la pedagogía, las personas se ponen en valor y pueden sentirse impulsadas a un proceso de emancipación singular y colectivo. 
Espacio íntimo de la pedagogía: relación educativa y su triple dimensión formativa como dinamismo de ciudadanía

Mónica GiJón CASARes

\section{Marco teórico: pedagogía y prostitución}

La relación educativa como espacio formativo no ha sido un tema de interés en los estudios de prostitución. Más bien, la prostitución femenina de carácter marginal ha sido abordada como fenómeno social por la antropología, la sociología y los estudios feministas desde muy diversas ópticas. Cabe destacar la caracterización de la industria del sexo y su diferenciación con otras realidades como la trata (Osborne, 2004; Agustín, 2004; Gijón, Cendón y Hernández, 2015); los trabajos que examinan las repercusiones personales y sociales del estigma en las mujeres (Pheterson, 2000; Juliano, 2002, 2006 y 2011); así como aquellos que profundizan en la conexión entre procesos migratorios y comercio sexual (Holgado, 2008; Agustín 2009).

De un tiempo a esta parte, son notables los trabajos que recogen experiencias y testimonios de mujeres en primera persona (Solana y Riopedre, 2012) e incluso aquellos que han sido elaborados por mujeres que ejercen la prostitución (Corso y Landi, 2000; Neira 2012). Todos ellos muestran la estrecha conexión entre dinamismos sociales y económicos que atraviesan la prostitución y cómo esta incide en la vida de las personas.

El trabajo socioeducativo en contextos de prostitución ha evolucionado en últimos veinte años. De abordajes de corte sanitario, enfocados en la salud sexual y la prevención de ETS, los proyectos trabajan ahora con ópticas más globales que contemplan aspectos psicológicos, jurídicos y socioeconómicos que afectan a las personas que ejercen la prostitución. Las instituciones multiplican sus ofertas formativas; diseñan programas de reducción de daño y educación de pares (Ballester, Orte y Perelló, 2004; Programa Sicar-Gijón, 2007); y, promocionan redes de apoyo mutuo, fortaleciendo el papel de las mujeres como sujetos políticos.

Como podemos observar, la prostitución como fenómeno social ha sido analizada en numerosos trabajos antropológicos y sociológicos. De igual modo, la intervención socioeducativa especializada en dicho ámbito ha sido descrita en diferentes propuestas pedagógicas. Sin embargo, cabe destacar que la relación como espacio formativo no ha sido abordada en profundidad en los trabajos sobre prostitución. Meneses (2000 y 2006) es pionera en mencionar la relación como elemento clave en el acompañamiento social, así como la importancia de las actitudes tole- 
rancia y respeto de los equipos en el trabajo con mujeres. No obstante, se trata de orientaciones tan amplias y genéricas que son válidas para cualquier ámbito educativo y no muestran la complejidad de la relación como una vía formativa.

\section{Marco empírico: acercarse a la relación en contextos de prostitución}

Visibilizar ese espacio íntimo de pedagogía requiere una mirada comprensiva que permita comprender el dinamismo de la relación en toda su complejidad. En el trabajo que nos ocupa, la etnometodología y investigación cualitativa de corte etnográfico nos ha permitido adentrarnos en la realidad del trabajo pedagógico con mujeres en situación de prostitución (Goetz y Lecompte, 1988; Coulon, 1995; Velasco y Díaz de la Rada, 1997; Geertz y Clifford, 2008; Angrosino, 2012). Investigaciones realizadas en Europa y América que nos han permitido hacer emerger el saber implícito y las acciones características de la intervención pedagógica. De toda la variedad y complejidad de prácticas pedagógicas, destaca la relación educativa por el hondo calado que deja en las personas que en ella participan.

Hemos analizado los dinamismos educativos que se dan en las instituciones, prestando especial atención al orden microsocial y a la red relacional que construyen educadoras y mujeres (Goffman, 1967 y 1997). Un trabajo que nos ha permitido analizar, interpretar y dotar de contenido los procesos dinámicos e íntimos de la pedagogía. Una aproximación cualitativa que parte de la palabra y la experiencia de las personas como fuente privilegiada de conocimiento, y que las reconoce como agentes capaces de explicar y transformar las condiciones que les envuelven. Por esa razón, hemos combinado metodologías etnográficas con trabajos narrativos e historias de vida que nos han ayudado a comprender la complejidad de la prostitución y el papel nuclear de la relación en los procesos formativos de las mujeres (Bertaux, 2004; Bolívar, Domingo y Fernández, 2001; Delory-Momberger, 2014).

El trabajo que aquí presentamos es fruto de varias investigaciones realizadas con mujeres en situación de prostitución y con profesionales que las acompañan en veinte instituciones en México, Italia, Reino Unido, Marruecos, Colombia, Bolivia y España desde 2009 hasta 
Espacio íntimo de la pedagogía: relación educativa y su triple dimensión formativa como dinamismo de ciudadanía

Mónica GiJón CASARes

$2016^{2}$. Algunas de esas instituciones son proyectos residenciales, otras son entidades formativas y otras de asesoría y seguimiento social, jurídico y médico. En dichas investigaciones hemos utilizado diferentes técnicas de observación participante, asesoramiento evaluativo, grupos de discusión, entrevistas y relatos de vida con mujeres que ejercen la prostitución y con profesionales (Taylor y Bogdan, 1984; Hammersley y Atkinson, 1994; Bertaux, 2004; Morriña, 2017).

Las técnicas utilizadas nos han permitido tener acceso a diferentes prácticas educativas de trabajo en el medio -calle, clubs y pisos privados donde se ejerce la prostitución-, principalmente, en entornos marginales; hemos observado prácticas de inserción laboral, de formación y capacitación técnica que se realizan en las diferentes instituciones; y, hemos compartido espacios convivenciales en casas de acogida y centros de escucha. Analizamos alrededor de noventa entrevistas a mujeres que ejercen o han ejercido la prostitución por algún tiempo-; cincuenta a profesionales especializadas -educadoras, formadoras, trabajadoras sociales, pedagogas y psicólogas-; y treinta grupos de discusión, grupos focales y dinámicas participativas, que nos han permitido reconocer saberes que provienen de la experiencia y sistematizar la relación como dinamismo formativo.

\section{Resultados y discusión: triple naturaleza formativa de la relación}

La relación es una vía de humanización que, en situaciones de exclusión social, responde a una triple naturaleza de encuentro, alianza y emancipación. No podemos reducir la importancia de la relación a la idea de acompañamiento, sino que nos encontramos ante el espacio educativo más íntimo y transversal de la práctica educativa. Un dina-

2 I+D, Mujer, inmigración y prostitución. Elementos para la intervención socioeducativa (Ref. EDU-2.008-04.724-E/EDUC; periodo 2009-2012 ); Instituto Catalan de la Mujer, Esclavitudes del s.XXI: trata de mujeres (Ref.U-7/08, periodo 2008-09), y Mujeres en situación de prostitución: intervención socioeducativa y participación (Ref.U/110-10; periodo, 2010-11); Ministerio de Igualdad, Mujeres en la frontera: prostitución y trata en el contexto de México (Ref.PACUI 11/2011/16, periodo: 2011-12); Convenio de investigación entre el GREM y el área Internacional de Acción Liberadora de la entidad RR.Adoratrices (América, Europa, Asia) Cultura Adoratriz y Pedagogía. Sistematización del modelo de atención a mujeres en contextos de exclusión, (Ref.Convenio de investigación, periodo 2013-2016). 
mismo que invita pensar la pedagogía de un modo más amplio que como vehículo de interconexión o transmisión de saberes. La relación puede describirse como una forma de entrar en relación, construir un espacio común e impulsar a la emancipación en el seno de una comunidad.

El potencial formativo de las relaciones se inicia con un primer dinamismo pedagógico que hemos denominado, entrar en relación. Una disposición al encuentro con la mujer y su realidad, que se da en un clima de acogida y se concreta en una invitación a realizar un proceso educativo. Entrar en relación permite a las mujeres realizar una experiencia educativa y, a la vez, experimentar un primer nivel de reconocimiento en las relaciones.

La cercanía resulta insuficiente para alcanzar el potencial formativo de la relación. La pedagogía requiere un segundo movimiento, que Ilamamos, construir un espacio común. Una alianza entre la mujer y la educadora que se da en un clima de cuidado y comprensión y se orienta a la autonomía. Lejos del conformismo y la neutralidad, la relación requiere de las educadoras tacto pedagógico y exigencia. De igual modo, la relación exige a las mujeres una actitud de confianza y participación. Actitudes nada fáciles cuando se han vivido situaciones de exclusión y cosificación.

Entrar en relación y comprometerse con la persona, no tendría carácter formativo si no estuviera acompañado de tercer movimiento, que denominamos, impulsar a la emancipación. Un dinamismo de reconocimiento que busca una mayor autorrealización e integración de las mujeres. Una orientación de las educadoras a reconocer logros y favorecer procesos de toma de conciencia que conduzcan a una mayor optimización. Una condición emancipadora que no se reduce un ámbito personal sino que motiva a la participación en la comunidad.

El siguiente cuadro refleja la triple naturaleza formativa de la relación que nos permite comprender la pedagogía como una forma de relación que se orienta al encuentro, la alianza y la emancipación. 
Espacio íntimo de la pedagogía: relación educativa y su triple dimensión formativa como dinamismo de ciudadanía

Mónica Gijón CASARES

\section{Pedagogía como relación}

La pedagogía puede concebirse como una forma de entrar en relación, construir un espacio común y impulsar a la emancipación en el seno de una comunidad.

\begin{tabular}{|c|c|c|}
\hline \multicolumn{3}{|c|}{ Naturaleza pedagógica de la relación } \\
\hline Entrar en relación & $\begin{array}{l}\text { Construir un espacio } \\
\text { común }\end{array}$ & $\begin{array}{l}\text { Impulsar a la emanci- } \\
\text { pación }\end{array}$ \\
\hline $\begin{array}{l}\text { Disposición al encuen- } \\
\text { tro con la mujer en un } \\
\text { marco de acogida para } \\
\text { invitar a realizar un } \\
\text { proceso educativo }\end{array}$ & $\begin{array}{l}\text { Disposición a una } \\
\text { alianza de cuidado, } \\
\text { comprensión y exigen- } \\
\text { cia con la mujer en el } \\
\text { marco de un trayecto } \\
\text { orientado a la autono- } \\
\text { mía }\end{array}$ & $\begin{array}{l}\text { Disposición al recono- } \\
\text { cimiento con carácter } \\
\text { emancipador para } \\
\text { invitar a un proceso de } \\
\text { integración personal y } \\
\text { social }\end{array}$ \\
\hline
\end{tabular}

*Cuadro elaboración propia

\section{Entrar en relación: disposición al encuentro}

Cuando una mujer que ejerce la prostitución llega al servicio de orientación de un proyecto social, siente una gran incertidumbre por lo va a encontrar y un profundo temor a ser reconocida como una mujer de la calle. La profesional que la recibe sabe de esos miedos y no la abruma con preguntas incómodas o normativas, más bien se esfuerza en proporcionar una acogida cálida. Cuando las educadoras visitan a calles, pisos y clubes donde se ejerce de prostitución su finalidad es orientar y repartir material preventivo, sin embargo, saludan a las mujeres, mantienen breves intercambios y se interesan por sus historias y proyectos. Una aceptación sincera en los primeros encuentros permite a las mujeres experimentar un primer nivel de reconocimiento en las relaciones. Así se muestra en el siguiente testimonio,

"lo más duro son los prejuicios, la crítica de la gente, además del miedo a que tu familia se entere y te rechace. En la prostitución te toca tolerar a clientes bien pesados, pero a veces son peor son las ofensas, el ser mal vista. A las sexoservidoras nos ven como una mujer de lo peor. Y puedo llegar a entender que quizá son personas ignorantes, pero nos ven paradas en la calle y les dicen a sus hijos, <pasa, no te acerques> y la verdad, te sientes mal. Como si estuvieras apestada, como te que les vas a pegar algo, rechazada de la sociedad" (Entrevista mujer, Proyecto CMA. RR. Oblatas, Distrito Federal-México). 
Como se aprecia en el testimonio, el estigma y el rechazo social son daños asociados a la prostitución que requieren una actitud respeto y trato digno con cada mujer. La prostitución tiene múltiples ópticas de análisis, sin embargo, es siempre un acontecimiento único en la historia de cada mujer. Por ese motivo, las educadoras se esfuerzan en abandonar las imágenes que desdibujan a la persona y tratan de escuchar cada realidad singular sin caer en tópicos.

La relación se inicia con una disposición al encuentro, una orientación a escuchar que se interesa más por la persona que por los motivos que la llevaron a la prostitución. Una disposición al tú que Buber (1998) describe como relación humanizante o relación yo-tu que, a diferencia de la relación objetivante y posesiva del yo-ello, solo es posible desde el amor o el diálogo. Se trata de escuchar y ponerse a disposición de cada mujer, antes que determinar demandas, necesidades o comprobar la veracidad de los datos de cada caso.

La disposición al encuentro se concreta en la acogida. Un recibimiento cálido que busca provocar una sensación de bienestar pero que, además, invita a realizar un proceso educativo (Rogers, 2002). Para algunas mujeres su paso por el proyecto no irá más allá de participar en un taller o una actividad de ocio, sin embargo, la invitación puede ser una oportunidad para abandonar sentimientos que la limitan, dotar de sentido su experiencia y trazar un proyecto vital encaminado a la autorrealización y la emancipación.

\section{Construir un espacio común: disposición a la alianza}

La relación educativa no se agota en el encuentro, para alcanzar su potencial formativo requiere de un espacio de compromiso y confianza que requiere tiempo. La realidad de las mujeres en situación de prostitución es muy diversa, sin embargo, muchas mujeres comparten la sensación de fracaso y decepción ante la falta de oportunidades. En los casos de trata, las mujeres experimentan, además, una sensación culpabilidad debido a la violencia física, el chantaje emocional y la falta de control sobre sus propias vidas. Huellas que no siempre son visibles, que conducen a una actitud de hermetismo y que requieren tacto pedagógico y tiempo para construir un espacio común. Así lo refiere el siguiente testimonio, 
Espacio íntimo de la pedagogía: relación educativa y su triple dimensión formativa como dinamismo de ciudadanía

Mónica GiJón CASARes

"es importante estar con ellas, estar presente, creo que es una cosa que las chicas captan y que les hace crear una relación, empezar a fiarse. Te das cuenta de cómo están, las miras, les preguntas cómo están. Y ellas se dan cuenta que las miras, que estás pendiente, aunque no digas nada. El ambiente familiar, o la vida cotidiana o qué se yo, pero cuando están preocupadas te das cuenta, y estás ahí. Es como crear una relación de iguales, ciertamente cada una con su rol, pero se trata de estar ahí. Y esto ellas lo captan" (Entrevista educadora, Proyecto Kairós-RR. Adoratrices, BérgamoItalia).

La relación es presencia y participación conjunta, requiere compartir espacios y construir lazos sólidos por medio del acompañamiento. En los proyectos, las responsabilidades comunes, las prácticas formativas o espacios de ocio consolidan la relación entre educadoras y mujeres.

Construir un espacio común es una disposición a la alianza que permite a la mujer sentirse apoyada y comprometida con su propio recorrido pedagógico. La alianza se concreta en un clima de cuidado y tiene una primera expresión de asistencia, donde la relación se torna más bien en un lugar de protección, un espacio donde la mujer pueda sentirse respetada y querida. Sin embargo, el cuidado no se queda en la preocupación, evoluciona hacia la exigencia, que invita a la mujer adquirir mayores cotas de autonomía. La alianza es una expresión de lo que Lévinas (1977 y 2012) denomina compromiso incondicional y asimétrico con el otro. Algo que Noddings (2009) señala al referirse a la condición relacional de la vida humana a partir de la interdependencia y de la necesidad de comprensión y afecto.

Construir un espacio común resulta exigente para mujeres y educadoras: ambas deben abrirse para entrar en un proceso de mutualidad. Una malla de interdependencia que permite a las mujeres realizar diferentes tránsitos hacia la autonomía, reconocer saberes y descubrirse capaz de afrontar nuevos retos y aspiraciones

\section{Impulsar a la emancipación: disposición al reconocimiento}

Cuando las mujeres Ilevan tiempo participando en actividades formativas y de asesoramiento en los proyectos sociales, son conscientes que hay acontecimientos en su historia más dolorosos incluso que la prosti- 
tución. A través de diferentes prácticas de clarificación como entrevistas, asambleas y tutorías, los equipos pueden acompañar procesos reflexivos y narrativos que permitan reconocerse y dotarse de sentido. Impulsar a la emancipación no es algo que las educadoras tengan que inocular, se trata más bien de un impulso de transformación y mejora, que es tan variado como la experiencia de las mujeres en la prostitución. En ocasiones, las mujeres lo encuentran en los hijos, en la necesidad reconstruir su proyecto vital o en la participación colectiva. Así lo refiere el siguiente testimonio.

"empecé a participar en cabildos y en consejos de juventud. Entonces noté que había como un liderazgo allí guardado y escondido, que también la gente empezó a notar. Me contrataron en la asociación como operadora par (...) es como estar en una escuela donde te forman y te pagan a la vez. Estás aprendiendo, aprendiendo y aprendiendo cosas; también a partir de todas tus experiencias positivas y negativas te das cuenta puedes ayudar a otras chicas, es un trabajo entre pares (...). Yo sé cómo es la historia, cómo hablarles desde mi lenguaje, qué viven las mujeres y cómo conectar con ellas" (Entrevista mujer líder transexual, Fundación PROCREAR, BogotáColombia).

Ayudar a otras mujeres que ejercen la prostitución, tiene un alto impacto en la autoestima. Con mujeres que han vivido situaciones de marginación y vulnerabilidad, la lógica de la donación tiene importantes efectos en la identidad: les permite situarse en otro lugar, sentir que su papel es importante y que su experiencia es útil y necesaria en la comunidad.

Impulsar la emancipación es una disposición al reconocimiento de la mujer que consiste, en creer en el valor de la persona y confiar en que cada mujer posee las herramientas necesarias para dirigir su vida. Para Honneth (2010) hay un primer nivel de reconocimiento que se produce en los vínculos sociales y que permite experimentar la autoconfianza. En ese sentido, la relación educativa no "da" algo que las mujeres no tengan, sino que pone en valor saberes y experiencias de la persona. Un reconocimiento que Ricoeur (2005) señala desde el ágape y el don, una aproximación más cercana a la idea de mutualidad.

Cuando el reconocimiento se orienta a la emancipación, la tarea de 
Espacio íntimo de la pedagogía: relación educativa y su triple dimensión formativa como dinamismo de ciudadanía

Mónica GiJón Casares

las educadoras no se limita a la mirada atenta, sino que pretende que las mujeres puedan sanar sus heridas, orientarse con inteligencia hacia sus deseos y ponerse en marcha con iniciativa para conseguirlos (Cyrulnik, 2005 y 2007). Sin embargo, la emancipación no tiene exclusivamente sentido individual de reconstrucción de sí, sino que posee una dimensión social cuando invita a las mujeres a contribuir en la comunidad y a tomar otro rol en la ciudadanía.

\section{Conclusiones}

La relación en contextos de prostitución posee una triple dimensión educativa que orienta al encuentro, la alianza y la emancipación de las mujeres. Las educadoras no se limitan a acompañarlas en su paso por las instituciones, sino que se disponen a la escucha y al trato digno, buscan estrechar un compromiso que ayude a la mujer a sacar lo mejor de sí misma y reconocen a las mujeres como capaces de dirigir su vida en la dirección que quieran.

La relación es un dinamismo formativo de alto impacto, un vehículo que se orienta a la autorrealización y que constituye una atmósfera de humanización y reconocimiento en las instituciones. La relación no es neutral en contextos de exclusión está llena de contenido y se produce en tres movimientos, entrar en relación, construir un espacio común e impulsar la emancipación. A modo de conclusión, veamos a continuación algunas consideraciones finales:

- La triple naturaleza de la relación no responde a una lógica jerárquica. No tiene más importancia el encuentro, la alianza o la emancipación en la relación, sino que los tres dinamismos operan con la misma significación en la pedagogía. Para una mujer tan importante es sentir que la educadora comprende su situación como experimentar que se ha comprometido con ella y reconoce sus logros y capacidades. La triple dimensión formativa es complementaria y logra, en conjunto, una experiencia transformadora de sí y del entorno.

- La dimensión formativa de la relación no responde a una lógica excluyente. Los dinamismos pedagógicos de la relación se combinan de forma dinámica y transversal al servicio de cada mujer, sin que sea necesario escoger entre el encuentro, la alianza o la 
emancipación. En contextos de prostitución, la pedagogía no debe conformarse con el cuidado y la asistencia de las mujeres, sino que debe impulsar a la toma de conciencia y la emancipación personal y colectiva.

- Una triple naturaleza de la relación puede ser válida para otros ámbitos. Estos tres dinamismos pedagógicos no tienen por qué reducirse a situaciones de prostitución, sino que pueden exportarse a otros ámbitos educativos. Una propuesta que nos ayuda a pensar la pedagogía más como proximidad humanizadora que como distancia profesional; más como construcción de un espacio común que como vehículo de reinserción o rescate; y más como un dinamismo de emancipación que como conquista solitaria de la autonomía.

\section{Referencias}

Angrosino, M. (2012) Etnografía y observación participante en investigación cualitativa. Madrid: Morata

Ballester, L., Orte, C. y Perelló, J. (2004). Programa de apoyo socioeducativo para mujeres jóvenes dedicadas a prostitución. Educació i Cultura: Universitat de les Illes Balears, 16, 105-112.

Barahona, M. J. y García, L. M. (2004). Manual de intervención social con mujeres prostituidas. Madrid: Dirección General de la Mujer..

Bertaux, D. (2005) Los relatos de vida. Perspectiva etnosociológica, Barcelona: Bellaterra.

Bolívar, A., Domingo, J., Fernández, M. (2001). La investigación biográfico-narrativa en educación. Enfoque y metodología. Madrid: La Muralla.

Búber, M. (1998). Yo y Tú. Madrid: Caparrós Editores.

Corso, C. y Landi, S. (2000). Retrato de intensos colores. Madrid: Talasa.

Coulon, A. (1995). Etnometodología y Educación. Barcelona: Paidós.

Cyrulnik, B. (2005) El amor que nos cura. Barcelona: Gedisa.

Cyrulnik, B. (2007) Las almas heridas. Barcelona: Gedisa.

Délory-Momberger. C. (2014) Autobiografía y educación: tradiciones, diálogos y metodologías. Revista Mexicana de Investigación Educativa, 3(62), 695-710.

Foucault, M. (2010) Vigilar y castigar. Madrid, S.XXI.

Goetz, J. P. y Lecompte, M.D. (1998). Etnografía y diseño cualitativo de la investigación educativa. Madrid: Morata.

Gijón, M. (2014). Trayectorias de mujeres en contextos de prostitución: saberes y fortalezas. En L. Faria, Y. Lima y P. Coelho. (2014), Histórias de vida, gênero e educaçâo. Curitiba-Brasil: CRV.

Gijón, M., Cendón, R. y Hernández, A. (2015). Trata de personas y Derechos Humanos: 
Espacio íntimo de la pedagogía: relación educativa y su triple dimensión formativa como dinamismo de ciudadanía

Mónica Gijón CASARES

Espacio íntimo de la pedagogía: relación educativa y su triple dimensión formativa como dinamismo de ciudadanía: retos y oportunidades desde la Educación Social. Revista de Educación Social, 20, 40-62.

Gijón, M. y Seguí, V. (2012). Aproximación a una pedagogía de la acogida y el reconocimiento. Experiencia de un camino compartido junto a las víctimas de trata. Madrid: Proyecto Esperanza y Sicar cat.

Gijón, M. (2017) Pedagogía Adoratriz. Una experiencia de amor, liberación y encuentro. Madrid: Adoratrices.

Goffman, E. (1970) El ritual de la interacción. Buenos Aires: Tiempo Contemporáneo

Goffman, E. (1997) La presentación de la persona en la vida cotidiana. Buenos Aires: Amorrortu.

Geertz, C.; Clifford, J. (2008) El surgimiento de la antropología posmoderna. Barcelona: Gedisa.

Hammersley, M., Atkinson, P. (1994) Etnografía. Métodos de investigación. Barcelona: Paidós.

Honneth, A. (2010) Reconocimiento y menosprecio. Sobre la fundamentación normativa de una teoría social. Madrid: Katz.

Honneth, A. (2011) La sociedad del desprecio. Madrid: Trotta.

Juliano, D. (2006) Excluidas y marginales. Madrid: Cátedra

Juliano, D. (2011) Presunción de inocencia. Riesgo, delito y pecado en femenino. Donostia: Gakoa.

Lévinas, E. (2000) Ética e infinito. Madrid: La balsa de la Medusa.

Lévinas, E. (2012) Totalidad e infinito. Salamanca: Sígueme.

Lévinas, E. (2001) Entre nosotros. Ensayos para pensar en otro. Valencia: Pre-Textos

Meneses, C. (2006) Actuación en el ámbito de la prostitución. En F. Vidal (Coord.), Exclusión social y estado del bienestar en España (pp. 553-560). Barcelona: Icaria.

Meneses, C. (2000). Trabajo social con prostitutas, en Martínez, M. J. (2000), Para el trabajo social: aportaciones teóricas y prácticas (pp. 449-470) Granada: Maristain.

Neira, M. (2012) Una mala mujer. La prostitución al descubierto. Barcelona: Plataforma.

Noddings, N. (2009) La educación moral. Propuesta alternativa para la educación del carácter. Buenos Aires: Amorrortu

Moriña, A. (2017) Investigar con historias de vida. Madrid: Narcea.

Orte, C. y March, M. (1998). Vers un model d'intervenció socioeducativa sobre la prostitució. Educació Social. Revista d'Intervenció Socioeducativa, 9.

Osborne, R. (2004). Trabajadoras del sexo. Derechos, migraciones y tráfico en el s.XXI. Barcelona: Bellaterra.

Planella, J. (2016) El acompañamiento social. Barcelona: UOC.

Pheterson, G. (2000) El prisma de la prostitución. Madrid: Talasa.

Pheterson, G. (2013) Mujeres en flagrante delito de independencia. Barcelona: Bellaterra.

Rogers, C. (2002) El proceso de convertirse en persona. Barcelona: Paidós.

Ricoeur, P. (2005) Caminos del reconocimiento. Madrid: Trotta.

Sicar-Gijón (2007) Multiplícate. Experiencia con mujeres inmigrantes que ejercen la prostitución. Gijón: Programa Sicar-RR.Adoratrices. 
Espacio íntimo de la pedagogía: relación educativa y su triple dimensión formativa como dinamismo de ciudadanía Mónica GiJón CASARes

Solana, J., Riopedre, J. (2012) Trabajando en la prostitución: doce relatos de vida. Granada: Comares.

Taylor, S. y Bogdan, R. (1984) Introducción a los métodos cualitativos de investigación. Barcelona: Paidós.

Velasco, H. y Díaz, R. (1997). La lógica de la investigación etnográfica. Madrid: Trotta. 
